\title{
Peptides going sweet: Synthesis of glycopeptide hybrid structures using Passerini and Ugi reactions
}

\author{
Andreja Jakas, Aleksandar Višnjevac, Ivanka Jerić
}

Rudjer Bošković Institute, Croatia

https://doi.org/10.17952/35EPS.2018.102

$\alpha$-Acyloxy and $\alpha$-acylamino carboxamides are the building blocks for the synthesis of various natural products, such as peptide and peptoid derivatives. Multicomponent reactions (MCRs) offer an attractive one-pot strategy for generating a library of these highly functionalized and complex organic compounds. The Passerini and Ugi reactions are isonitrile-based MCR that yields $\alpha$-acyloxy and $\alpha$-acylamino carboxamides, respectively (Scheme1). Passerini reaction involves an aldehyde, an isonitrile, and a carboxylic acid, while Ugi reaction involves an aldehyde, an isonitrile, a carboxylic acid and an amine component.[1]The utilization of carbohydrates and their derivatives in MCRs is highly desirable, owing to their polyfunctional character and stereochemical diversity.[2]
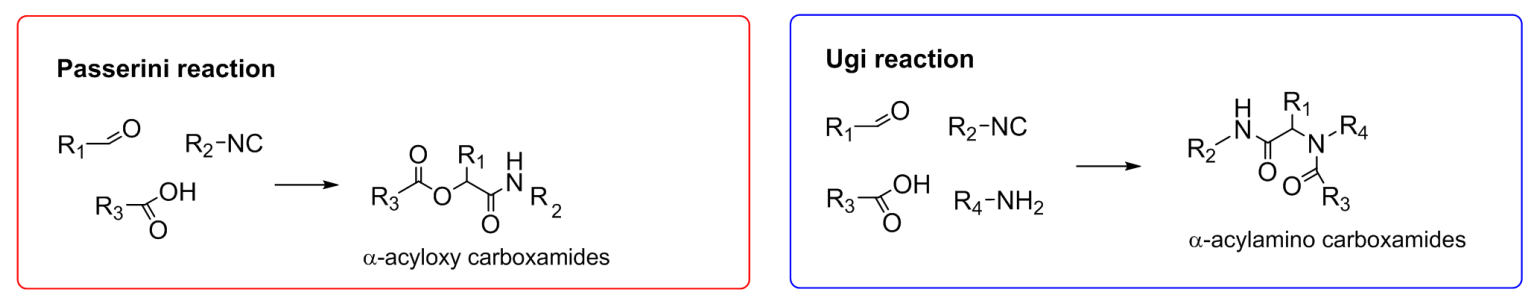

Scheme 1: Reactants and products of Passerini and Ugi reaction.

Our efforts were directed toward exploitation of carbohydrate derivatives as components in the Passerini and Ugi reactions. We used for the first time highly strained carbohydrate-derived aldehydes, isocyanides and amines in the Passerini and Ugi reaction with different commercially available carboxylic acids. As acidic non-sugar components, acetic acid, benzoic acid and Boc-protected phenylalanine were used, and as a sugar component isopropylidene protected gulonic acid (GulA) was used (Scheme 2). Three different sugar moieties were used as isopropylidene protected carbohydrate-derived components (aldehydes, isocyanide, and amine) in the Passerini and Ugi reaction: fructose (Fru), galactose ( $\mathrm{Gal}$ ) and sorbose (Sor). Combination of these components gave eleven (1-11) Passerini products (Scheme 3) and nine (12-20) Ugi products (Scheme 4).The prepared Passerini products bear up to three sugar components, while Ugi products comprise up to four sugar components.

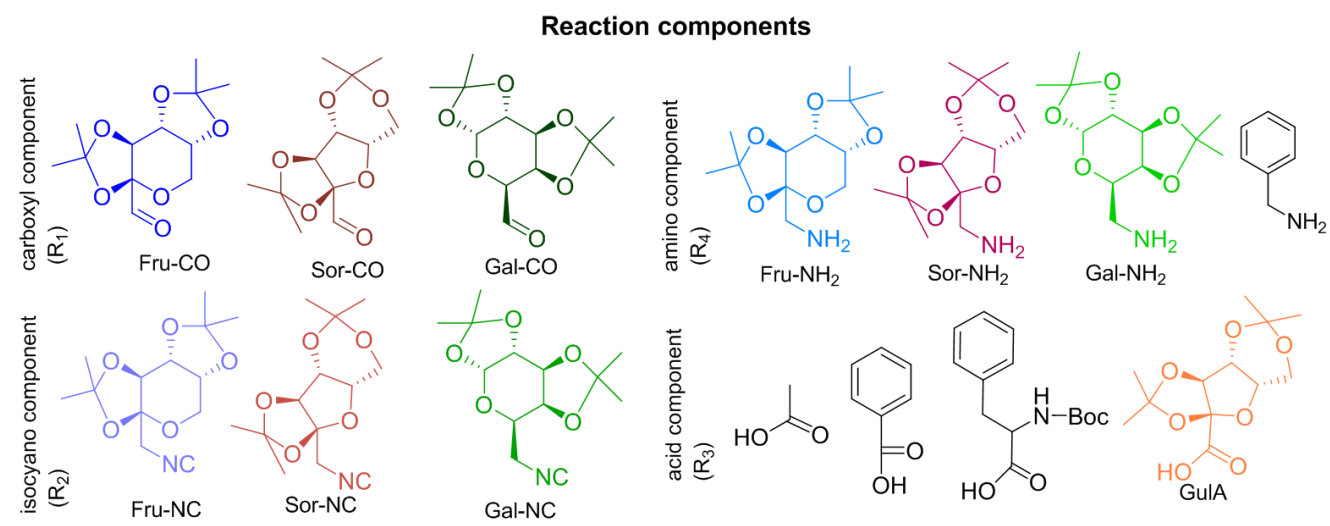

Scheme 2: Used isocyano, carboxyl, amino and acid components in Passerini and Ugi reactions.

The Passerini reactions conducted with prepared sugar aldehydes were highly diastereoselective, with d.r. 9:1, and the crystal structure analysis revealed that the stereochemistry of newly formed chiral center is $\mathrm{S}$. The diastereoselectivity of Ugi reactions depends on component's structure and varies from d.r. 1:1 to d.r. 9:1 with $S$ isomer being predominant one. 

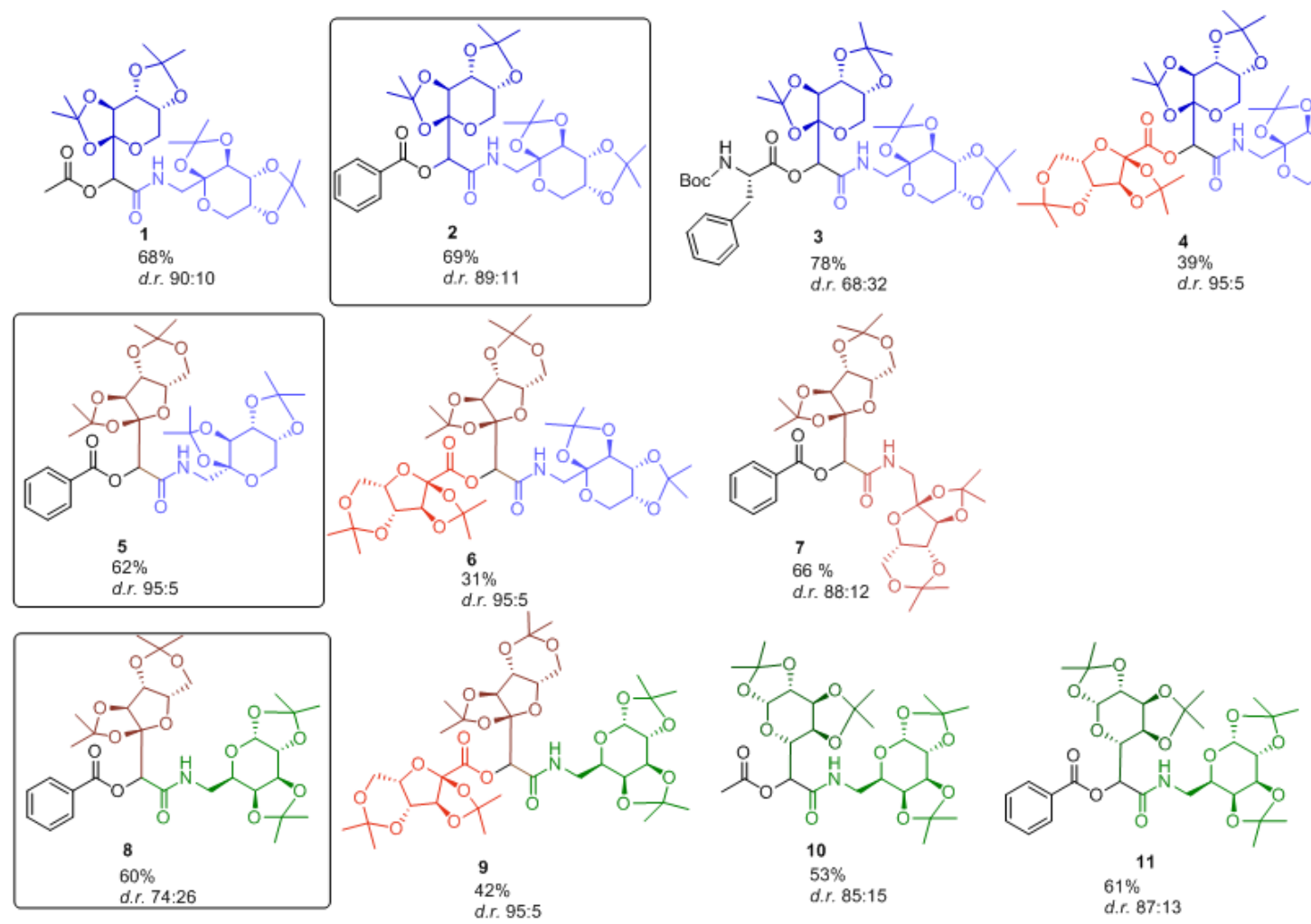

Scheme 3: Prepared Passerini reaction products. Crystal structures were determined for compounds in squares.

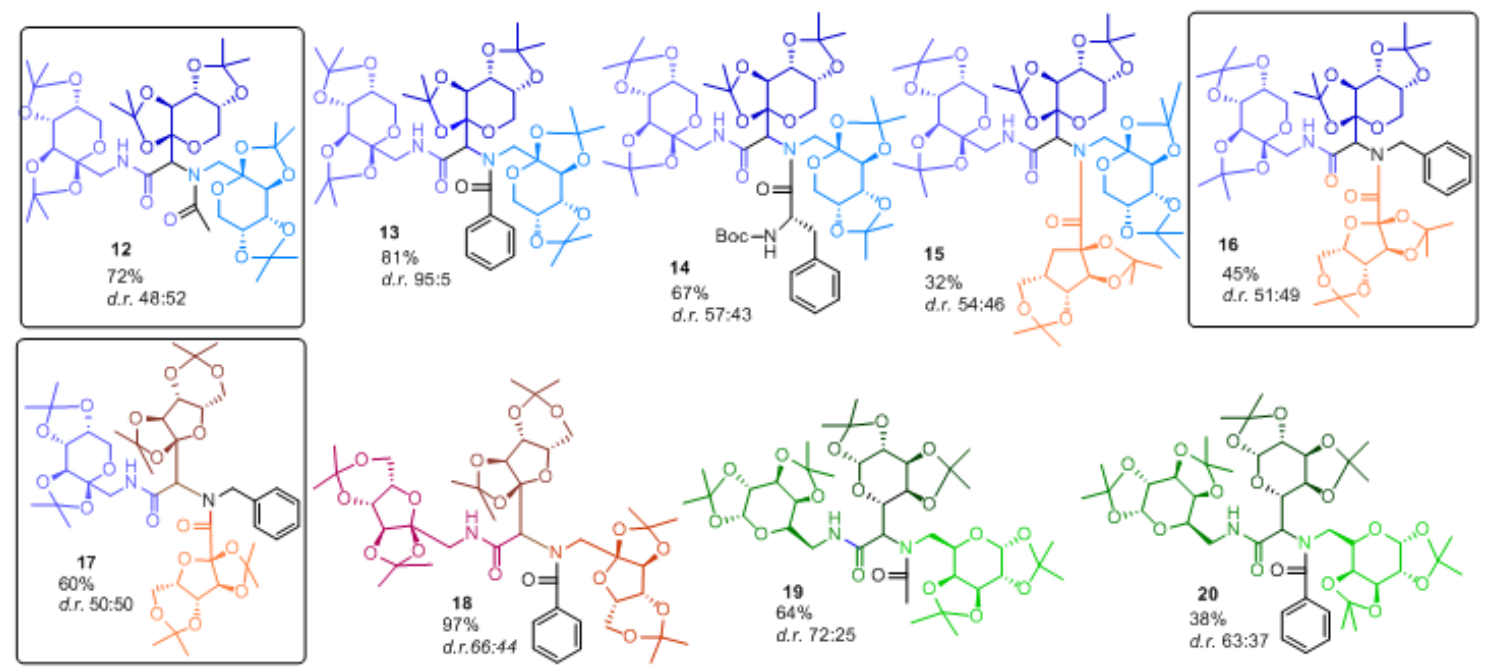

Scheme 4: Prepared Ugi reaction products. Crystal structures were determined for compounds in squares.

\section{Acknowledgements}

We are grateful for financial supportfrom the Croatian Science Foundation, Grant number 3102.

\section{References}

1. D. Fürniss et al. Beilstein J. Org. Chem. 2013, 9, 56-63.

2. Md. M. Khan et al. RSC Adv. 2015, 5, 57883-57905. 